\title{
PULSE-STREAM MODELS IN TIME-OF-FLIGHT IMAGING
}

\author{
Adrien Besson ${ }^{\star}$, Dimitris Perdios ${ }^{\star}, Y_{v e s}$ Wiaux* $^{*}$ Jean-Philippe Thiran ${ }^{\star}, \dagger$ \\ ${ }^{\star}$ Signal Processing Laboratory (LTS5), Ecole Polytechnique Fédérale de Lausanne, Switzerland \\ *Institute of Sensors, Signals and Systems, Heriot-Watt University, UK \\ ${ }^{\dagger}$ Department of Radiology, University Hospital Center and University of Lausanne, Switzerland
}

\begin{abstract}
This paper considers the problem of reconstructing raw signals from random projections in the context of time-of-flight imaging with an array of sensors. It presents a new signal model, coined as multi-channel pulse-stream model, which exploits pulse-stream models and accounts for additional structure induced by inter-sensor dependencies. We propose a sampling theorem and a reconstruction algorithm, based on $\ell_{1}$-minimization, for signals belonging to such a model. We demonstrate the benefits of the proposed approach by means of numerical simulations and on a real nondestructive-evaluation application where the peak-signal-to-noiseratio is increased by $3 \mathrm{~dB}$ compared to standard compressed-sensing strategies.
\end{abstract}

Index Terms - Compressed sensing, sparsity, array imaging

\section{INTRODUCTION}

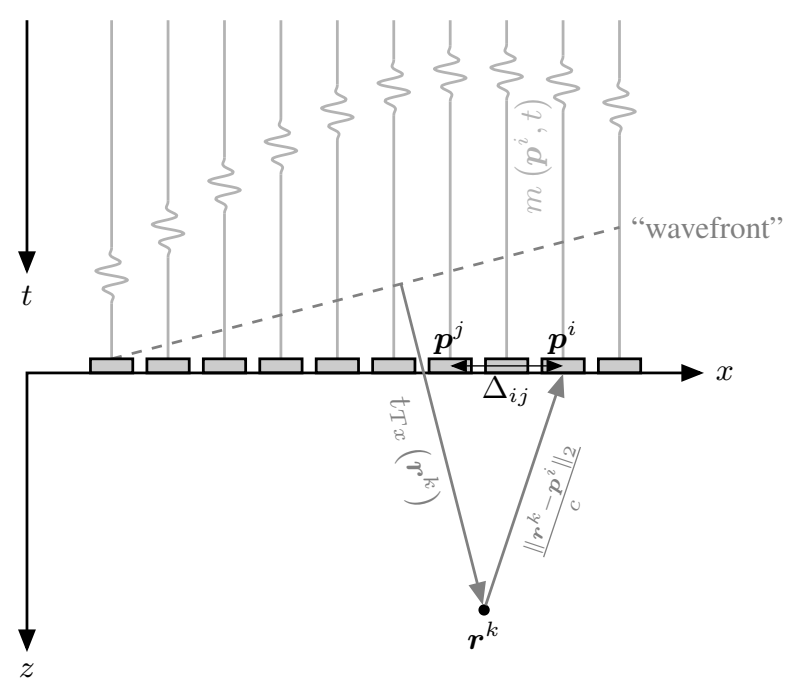

Fig. 1. Considered time-of-flight imaging configuration.

The notion of pulse stream has been introduced by Hedge and Baraniuk [1] and designates signals that can be expressed as a convolution between a $K$-sparse spike train and a $F$-sparse impulse response.

This work was supported by the UltrasoundToGo RTD project (no. 20NA21_145911), funded by Nano-Tera.ch.
Formally, let us consider a pulse stream $\boldsymbol{z} \in \mathbb{R}^{N}$, such that $\boldsymbol{z}=\boldsymbol{h} * \boldsymbol{s}$ with $\boldsymbol{s} \in \mathbb{R}^{N}$ the $K$-sparse spike train and $\boldsymbol{h} \in \mathbb{R}^{N}$ the $F$-sparse impulse response. The following definition holds:

Definition 1 (Definition 2 of [1]). The pulse-stream model is defined as follows:

$$
\mathcal{M}_{K, F}^{z}:=\left\{\boldsymbol{z} \in \mathbb{R}^{N}: \boldsymbol{z}=\boldsymbol{s} * \boldsymbol{h} \mid \boldsymbol{s} \in \mathcal{M}_{K} \text { and } \boldsymbol{h} \in \mathcal{M}_{F}\right\},
$$

where $\mathcal{M}_{K} \subset \mathbb{R}^{N}$ and $\mathcal{M}_{F} \subset \mathbb{R}^{N}$ are restricted unions of $L_{K}$ $K$-dimensional and $L_{F} F$-dimensional canonical subspaces, respectively.

For signals belonging to the pulse-stream model $\mathcal{M}_{K, F}^{z}$, Hedge and Baraniuk [1] have derived a sampling theorem where the number of measurements necessary for perfect reconstruction scales linearly with $K+F$ instead of $K F$ (standard CS). In this work, we propose to extend this model to time-of-flight imaging with an array of sensor elements, whose configuration is described on Fig. 1. The sensing process is divided into a transmit phase where one or several emitters are used to send a pulsed-wave in the medium, and a receive phase where the sensors are used to acquire the response of the medium to the previously transmitted pulsed wave. Such a configuration covers a wide range of applications such as medical ultrasound imaging, non-destructive evaluation, seismic imaging, sonar, lidar and synthetic aperture radar imaging.

Formally, let us assume that the array is made of $N_{e l}$ sensors, positioned at $\left(\boldsymbol{p}^{i}\right)_{i=1}^{N_{e l}}$, as described on Fig. 1. Let us also consider that the medium is made of $K$ targets positioned at $\left(\boldsymbol{r}^{k}\right)_{k=1}^{K}$. The signal $m_{i}(t)$ received at the $i$-th sensor can be expressed as:

$$
m_{i}(t)=\sum_{k=1}^{K} a_{k} h\left(t-t_{i}^{k}\right)
$$

where $a_{k}$ and $t_{i}^{k}$ are the amplitude and delay associated with the $k$-th target and $h(t)$ is the received pulse, supposed to be known in the remainder of the paper. The delay associated with the $k$-th target depends on its relative position with respect to the $i$-th sensor and can be expressed as follows:

$$
t_{i}^{k}=t_{T x}\left(\boldsymbol{r}^{k}\right)+\frac{\left\|\boldsymbol{r}^{k}-\boldsymbol{p}^{i}\right\|_{2}}{c},
$$

where $c$ denotes the wave velocity in the medium, supposed to be constant, and $t_{T x}\left(\boldsymbol{r}^{k}\right)$ is the transmit delay which depends on the transmit settings. Such model have been extensively used in medical ultrasound imaging [2, 3, 4], non-destructive testing [5] and radar imaging $[6,7]$. 
Starting from Equation (2), we consider inter-sensor dependencies in order to derive an additional structure of the array signals. This structure, expressed as restrictions on the possible support of the array signals, leads us to define a new model, denoted as multichannel pulse stream model, from which we present a sampling theorem and a recovery algorithm.

The remainder of the paper is organized as follows. In Section 2, the signal model is presented, with the corresponding sampling theorem and recovery algorithm. Section 3 presents results on synthetic pulse streams as well as on real non-destructive evaluation signals. Concluding remarks are given in Section 4.

\section{PULSE STREAMS IN ARRAY IMAGING}

\subsection{Signal recovery from the pulse-stream model}

From Equation (2), one may express the signal $m_{i}(t)$ as $m_{i}(t)=$ $\left(s_{i} * h\right)(t)$, where $h(t)$ is the pulse and

$$
s_{i}(t)=\sum_{k=1}^{K} a_{k} \delta\left(t-t_{i}^{k}\right) .
$$

Let us consider that the signal $m_{i}(t)$ is sampled at a rate $f_{s}$, leading to $N$ samples $m_{i}\left(t^{j}\right)$, with $t^{j}=t^{0}+j / f_{s}$ for $j \in\{1, \ldots, N\}$.

The vector $\boldsymbol{m}_{i}=\left[m_{i}\left(t^{1}\right), \ldots, m_{i}\left(t^{N}\right)\right]^{T} \in \mathbb{R}^{N}$ belongs to the pulse-stream model $\mathcal{M}_{K, F}^{z}$ where $F$ denotes the size of the support of $\boldsymbol{h} \in \mathbb{R}^{N}$, supposed to be small compared to $N$, and $K$ the number of point scatterers.

Thus, one may be able to sample array signals at a rate dictated by Hedge and Baraniuk [1] while ensuring a perfect recovery using either model-based greedy approaches [8] or $\ell_{1}$-minimization [9]. In the proposed work, we have decided to focus on the latter. Since the pulse is supposed to be known, the following convex problem can be solved to retrieve $\boldsymbol{m}_{i} \in \mathbb{R}^{M}$ from noisy measurements $\boldsymbol{y}=$ $\Phi \boldsymbol{m}_{i}+\boldsymbol{n}$, with $\Phi \in \mathbb{R}^{M \times N}$ a Gaussian i.i.d. matrix:

$$
\min _{\overline{\boldsymbol{s}}}\|\overline{\boldsymbol{s}}\|_{1} \text { subject to }\|\boldsymbol{y}-\Phi \mathrm{H} \overline{\boldsymbol{s}}\|_{2} \leq \epsilon,
$$

where $\mathrm{H}$ is a circulant matrix which contains time-shifted replicas of the pulse, $s$ is a $K$-sparse vector and $\epsilon \in \mathbb{R}_{+}$.

\subsection{Multi-channel pulse-stream model}

The model described in Section 2.1 is suited to single channel reconstructions. However, such a model does not account for interchannel dependencies, which are self-evident in the proposed configuration (see Fig. 1). By taking into account the dependencies, one may be able to decrease the number of measurements required to reconstruct array signals. The following theorem precises the way the dependencies between two channels may be expressed.

Theorem 1 (Two-channel scenario). The support $\sigma\left(s_{i}\right)$ of the spike train $\boldsymbol{s}_{i}$ corresponding to the sensor located at a distance $\Delta_{i j}$ from the sensor $j$, whose spike train is $\boldsymbol{s}_{j}$, has the following property:

$$
\sigma\left(\boldsymbol{s}_{i}\right) \subset S_{i j},
$$

where $S_{i j}:=\bigcup_{k=1}^{K} \Omega_{k}^{i j}$ is a union of $2 D_{i j}$-dimensional subspaces $\Omega_{k}^{i j}$ defined by:

$$
\Omega_{k}^{i j}:=\left\{\left\{k-D_{i j}, \ldots, k+D_{i j}\right\}, k \in \sigma\left(\boldsymbol{s}_{j}\right)\right\},
$$

where $D_{i j}=\left\lceil f_{s} \Delta_{i j} / c\right\rceil$.
In the above theorem, $\lceil$.$\rceil designates the round value.$

Proof. Let us suppose that $s_{j}(t)=\sum_{k=1}^{K} a_{k} \delta\left(t-t_{j}^{k}\right)$ and $s_{i}(t)=\sum_{k=1}^{K} a_{k} \delta\left(t-t_{i}^{k}\right)$. From Equation (3), one may deduce the following:

$$
\begin{aligned}
t_{j}^{k} & =t_{T x}\left(\boldsymbol{r}^{k}\right)+\frac{\left\|\boldsymbol{r}^{k}-\boldsymbol{p}^{j}\right\|_{2}}{c} \\
& \leq t_{T x}\left(\boldsymbol{r}^{k}\right)+\frac{\left\|\boldsymbol{r}^{k}-\boldsymbol{p}^{i}\right\|_{2}}{c}+\frac{\Delta_{i j}}{c} \\
& \leq t_{i}^{k}+\frac{\Delta_{i j}}{c} .
\end{aligned}
$$

Reversely, one can deduce that $t_{j}^{k} \geq t_{i}^{k}-\frac{\Delta_{i j}}{c}$, which leads to $t_{i}^{k} \in$ $\left[t_{j}^{k}-\frac{\Delta_{i j}}{c}, t_{j}^{k}+\frac{\Delta_{i j}}{c}\right]$. Thus, by simple multiplication with $f_{s}$, one may deduce that:

$$
\forall l \in \sigma\left(\boldsymbol{s}_{i}\right), \exists p \in \sigma\left(\boldsymbol{s}_{j}\right) \mid l \in\left\{p-D_{i j}, \ldots, p+D_{i j}\right\},
$$

where $D_{i j}=\left\lceil f_{s} \Delta_{i j} / c\right\rceil$. Generalizing Equation (6) to the support of $\sigma\left(\boldsymbol{s}_{i}\right)$, one may retrieve the result of Theorem 1 .

Theorem 1 states that the support of $\boldsymbol{s}_{i}$ is a union of $K 2 D_{i j}$ dimensional subspaces located around the support of the signal received at sensor $j$. The dimension of each subspace depends on the distance between the sensors.

We can go further than the two-channel scenario by considering that we have prior knowledge on multiple channels. In this case, the following theorem holds.

Theorem 2 (Multi-channel scenario). The support $\sigma\left(\boldsymbol{s}_{i}\right)$ of the spike train $\boldsymbol{s}_{i}$ corresponding to the sensor located at distances $\left(\Delta_{i j}\right)_{j=1}^{N}$ from a set of $N$ sensors, whose spike trains are $\left(\boldsymbol{s}_{j}\right)_{j=1}^{N}$, has the following property:

$$
\sigma\left(\boldsymbol{s}_{i}\right) \subset S
$$

where $S:=\bigcap_{j=1}^{N} S_{i j}$ is the intersection of the spaces $S_{i j}$ defined in Theorem 1.

Proof. This is a simple generalization of Theorem 1. Let us denote as $\left(\boldsymbol{s}_{j}\right)_{j=1}^{N}$ the spike trains associated with the $N$ considered sensors. Then, Theorem 1 states that:

$$
\forall j \in\{1, \ldots, N\}, \sigma\left(\boldsymbol{s}_{i}\right) \in S_{i j} \Leftrightarrow \sigma\left(\boldsymbol{s}_{i}\right) \in \bigcap_{j=1}^{N} S_{i j}
$$

In this case, the support $\sigma\left(\boldsymbol{s}_{i}\right)$ is included into a smaller subspace, taking into account the dependencies between the sensor $i$ and the $N$ other ones. We use the result of Theorem 2 to define the multi-channel pulse-stream model as:

$$
\mathcal{U}_{K, F}^{z}:=\left\{\boldsymbol{z} \in \mathbb{R}^{N}: \boldsymbol{z}=\boldsymbol{s} * \boldsymbol{h} \mid \boldsymbol{s} \in \mathcal{M}_{K}, \sigma(s) \subset S\right\},
$$

where the pulse $\boldsymbol{h}$ is supposed to be known. 


\subsection{Sampling theorem for multi-channel pulse-stream signals}

The multi-channel pulse-stream model has an additional structure compared to the single-channel pulse-stream model, i.e. $\mathcal{U}_{K, F}^{z} \subset$ $\mathcal{M}_{K, F}^{z}$. This can be exploited in order to reduce the sampling rate requirements for signals belonging to $\mathcal{U}_{K, F}^{z}$. The theorem hereafter makes this precise and sets the sampling requirement.

Theorem 3. Suppose that $\mathcal{U}_{K, F}^{z}$ is the multi-channel pulse-stream model defined in Equation (7). Let $t>0$ and $\delta>0$. Choose a $M \times N$ i.i.d. Gaussian matrix $\Phi$ with

$$
M \geq \mathcal{O}\left((K+F) \ln \left(\frac{1}{\delta}\right)+K\left(1+\log \left(\frac{|S|}{K}\right)\right)+t\right) .
$$

Then $\Phi$ satisfies the following property with probability $1-e^{-t}$ $\forall \boldsymbol{z}_{1}, \boldsymbol{z}_{2} \in \mathcal{U}_{K, F}^{z}$,

$$
(1-\delta)\left\|\boldsymbol{z}_{1}-\boldsymbol{z}_{2}\right\|^{2} \leq\left\|\Phi \boldsymbol{z}_{1}-\Phi \boldsymbol{z}_{2}\right\|^{2} \leq(1+\delta)\left\|\boldsymbol{z}_{1}-\boldsymbol{z}_{2}\right\|^{2} .
$$

In the theorem above $|S|$ denotes the cardinality of the set $S$.

Proof. The proof is based on Theorem 1 of [1]. Suppose that $z \in$ $\mathcal{U}_{K, F}^{z}$, then, $\boldsymbol{z} \in \mathcal{M}_{K, F}^{z}$. From [1], one may set the bound $M$ as:

$$
M \geq \mathcal{O}\left((K+F) \ln \left(\frac{1}{\delta}\right)+\log \left(L_{K} L_{F}\right)+t\right)
$$

where $t>0$. When $\boldsymbol{h}$ is known, $L_{F}=1$. Moreover, if we consider that $\sigma(s) \subset S$, then the following inequality holds:

$L_{K} \leq\left(\begin{array}{c}|S| \\ K\end{array}\right) \approx\left(\frac{e|S|}{K}\right)^{K} \Leftrightarrow \log \left(L_{K}\right) \leq K\left(1+\log \left(\frac{|S|}{K}\right)\right)$

Introducing the above results in Equation (8) leads to the results of Theorem 3.

The main benefit of Theorem 3 is that the number of measurements required for perfect reconstruction are $\mathcal{O}(K \log (|S| / K))$, instead of $\mathcal{O}(K \log (N / K))$ in the case of the single-channel pulse stream model. Interestingly, such a reduction can be interpreted in light of the D-RIP property introduced by Candes et al. [9]. Indeed, a signal $z \in \mathcal{U}_{K, F}^{z}$ is $K$-sparse in the convolutional (coherent) dictionary $\mathrm{H}$, and can be acquired with $\mathcal{O}(K \log (N / K))$ Gaussian i.i.d. measurements [9]. In addition, $\boldsymbol{z} \in \mathcal{U}_{K, F}^{z}$ implies that $\sigma(s) \in S$ which means that the recovery problem can be solved in $\mathbb{R}^{|S|}$ rather than $\mathbb{R}^{N}$ and, consequently, that the signal can be acquired with $\mathcal{O}(K \log (|S| / K))$ Gaussian i.i.d. measurements.

\subsection{Recovery of multi-channel pulse-stream signals}

As described in Section 2.1, the signal $\boldsymbol{m}=\boldsymbol{s} * \boldsymbol{h}, \boldsymbol{m} \in \mathcal{U}_{K, F}^{z}$ can be written as $\boldsymbol{m}=\mathrm{H} \boldsymbol{s}$. Let us consider that the signal $\boldsymbol{y}=$ $\Phi \boldsymbol{m}$ is measured, where $\Phi \in \mathbb{R}^{M \times N}$ satisfies the requirements of Theorem 3. As stated in Section 2.3, the recovery problem in $\mathbb{R}^{N}$ can be recast as the following recovery problem in $\mathbb{R}^{|S|}$ :

$$
\text { Find } \boldsymbol{\alpha} \in \mathbb{R}^{|S|} \text { such that }\left\|\boldsymbol{y}-(\Phi \mathrm{H})_{\mid S} \boldsymbol{\alpha}\right\|_{2} \leq \epsilon,\|\boldsymbol{\alpha}\|_{0} \leq K,
$$

where $\epsilon \in \mathbb{R}_{+}$accounts for the noise level and $(\Phi \mathrm{H})_{\mid S} \in \mathbb{R}^{M \times|S|}$ corresponds to a submatrix of $\Phi \mathrm{H}$ formed by the columns indexed by the support $S$. Depending on the ratio between the number of measurements $M$, the size of the support $S$ and the noise level, two different recovery procedures may be considered.

\subsubsection{Recovery by least-square minimization}

When $M \geq|S|$ and $\epsilon=0$, Problem (9) involves an overcomplete matrix $(\Phi \mathrm{H})_{\mid S} \in \mathbb{R}^{M \times|S|}$ and can be solved by simple least-square minimization. In this case, the solution $\boldsymbol{\alpha}^{\star}$ of Problem (9) is expressed as:

$$
\boldsymbol{\alpha}^{\star}=(\Phi \mathrm{H})_{\mid S}^{\dagger} \boldsymbol{y},
$$

where $(\Phi \mathrm{H})_{\mid S}^{\dagger}$ denotes the Moore pseudo-inverse of $(\Phi \mathrm{H})_{\mid S}$.

\subsubsection{Recovery by $\ell_{1}$-minimization on the signal support}

In a more general case, $\boldsymbol{\alpha}^{\star}$ can be recovered by solving the following convex optimization problem [9]:

$$
\min _{\boldsymbol{\alpha} \in \mathbb{R}^{|S|}}\|\boldsymbol{\alpha}\|_{1} \text { subject to }\left\|\boldsymbol{y}-(\Phi \mathrm{H})_{\mid S} \boldsymbol{\alpha}\right\|_{2} \leq \epsilon .
$$

In the remainder of the paper, Problem (11) is solved using the alternating direction methods of multipliers (ADMM) [10].

\section{EXPERIMENTS}

We now present the results of experiments that validate the proposed approach and show its benefits.

\subsection{Synthetic pulse streams}

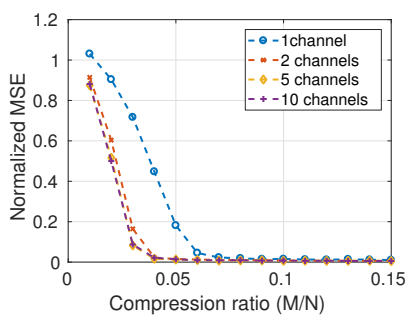

(a)

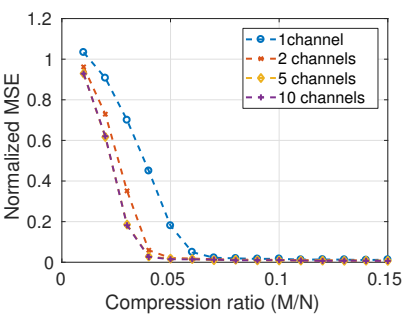

(b)
Fig. 2. Normalized MSE for (a) $\Delta=0.31 \mathrm{~mm}$ (one wavelength) and (b) $\Delta=0.62 \mathrm{~mm}$ (two wavelengths) vs. the compression ratio $(M / N)$ for the proposed method for 1-, 2-, 5- and 10-channel scenarios. Signals parameters: $N=2000, F=31, K=20$.

We consider a synthetic configuration with $K=20$ pointscatterers with random amplitudes and positions are generated. 10 sensors are considered, with an inter-sensor spacing of $\Delta$. Pulsestreams of length $N=2000$ are simulated mimicking ultrasound plane-wave imaging with normal incidence [11]. The considered pulse $h(t)$ is a convolution between a 2-cycle square excitation signal and a Gaussian pulse $(2.5$ cycles, center frequency $5.208 \mathrm{MHz}$, bandwidth $67 \%$ ) which mimics the impulse response of ultrasound transducer elements. The sampling frequency $f_{s}$ is set to $20.8 \mathrm{MHz}$ which corresponds to four times the center frequency.

Fig. 2 displays the averaged results of a Monte-Carlo simulation over 1000 trials of the ADMM algorithm. Each trial was conducted by randomly generating the amplitudes and positions of the $K$ point-scatterers, the Gaussian i.i.d. matrix $\Phi \in \mathbb{R}^{M \times N}$ and by reconstructing the raw data $\boldsymbol{m}$ of one sensor from different values of $M / N$. single-channel as well as multi-channel scenarios are considered. For the multi-channel scenarios, prior knowledge on the support of the spike trains of 1,4 and 9 neighboring sensors of the sensor of interest are considered. Figure 2(a) and 2(b) 


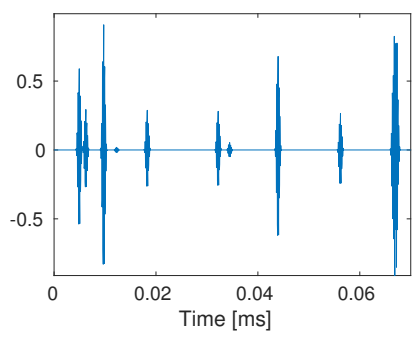

(a)

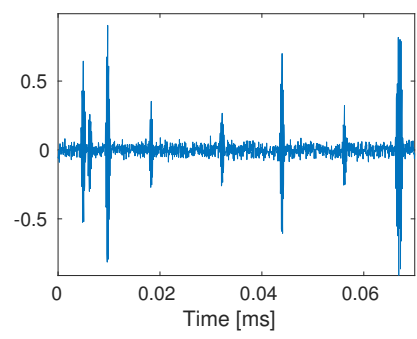

(b)

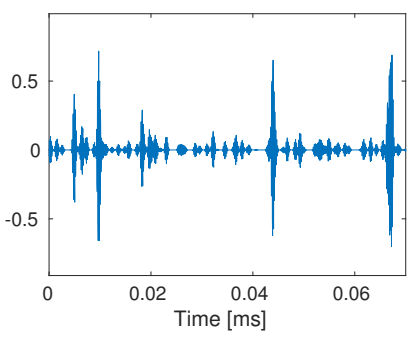

(c)

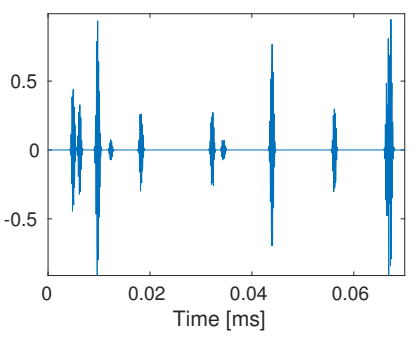

(d)

Fig. 3. (a) Original signal (b) Noisy signal $(\mathrm{SNR}=30 \mathrm{~dB})$ (c) Recovered estimate from $M=160$ measurements in a 1-channel scenario (d) Recovered estimate from $M=160$ measurements in a 5-channel scenario.

show the normalized mean squared error (NMSE), calculated as $\left\|\boldsymbol{m}-\boldsymbol{m}^{\star}\right\|_{2} /\|\boldsymbol{m}\|_{2}$, where $\boldsymbol{m}$ is the reference and $\boldsymbol{m}^{\star}$ the estimate, for two different inter-sensor spacings, namely $0.31 \mathrm{~mm}$ (one wavelength) and $0.62 \mathrm{~mm}$ (two wavelengths). Regarding the optimization algorithm, the maximum number of iterations is set to 1000 and $\epsilon=0$. This experiment also demonstrates that considering a higher number of channels, which decreases the dimension of the subspace $S$ (Theorem 2), results in a better recovery. Indeed, Fig. 2(a) show that the 10- and 5-channel scenarios outperform the 2-channel one.

Figure 3 shows that the proposed algorithm is robust to small amount of noise $(\mathrm{SNR}=30 \mathrm{~dB})$. For this experiment, a small amount of Gaussian noise is added to the element raw-data of each sensor, leading to the signal displayed on Figure 3(b). Figures 3(c) and 3(d) show the recovered signals for the 1-channel and 5-channels scenarios, respectively, for a number of measurements $M=160$. It can be seen that the signal recovered from the 5-channel scenario is closer to the original signal than the one recovered from the 1-channel scenario.

\subsection{Experimental non-destructive-evaluation signals}

An aluminum block containing side drilled holes located at different depths have been insonified with 1 plane wave (normal incidence) using an open phased-array platform (OEM-PA, Advanced OEM Solutions, Cincinnati, Ohio, USA), equipped with a linear probe (Imasonic SAS, Voray-sur-l'Ognon, France) composed of 64 elements with $0.93 \mathrm{~mm}$ pitch, working at $5 \mathrm{MHz}$ with $100 \%$ bandwidth. The sampling frequency has been set to $50 \mathrm{MHz}$ and the speed of sound in aluminum is $6300 \mathrm{~m} \mathrm{~s}^{-1}$. After acquisition, the element raw-data are imported on MATLAB and compressed using a Gaussian i.i.d. matrix $\Phi \in \mathbb{R}^{M \times N}$, with a compression ratio $M / N=0.03(7.5 \%$ of the Nyquist frequency). The pulse is approximated as a convolution between a 0.5 -cycle excitation signal and a 1-cycle Gaussianmodulated sinusoidal impulse response. In the 1-channel scenario, all the channels are reconstructed in parallel. In the multi-channel scenario, a sequential reconstruction is achieved where each channel is recovered with prior knowledge on the support of the spike train corresponding to the neighbouring channel (obtained from the previous reconstruction). The first channel is reconstructed from a compression ratio $M / N=0.5$ in order to have a relevant first estimation of the support of the spike train. Concerning the optimization algorithm, the maximum number of iterations is set to 1000 and $\epsilon=0.3\|\boldsymbol{y}\|_{2}$. Once the raw data are reconstructed from compressed measurements, standard delay-and-sum beamforming [11] is applied to generate the radio-frequency image. The envelope is extracted through Hilbert transform and normalized to obtain the B-mode im- age. Figure 4(a) displays the reference B-mode image obtained with no compression and Fig. 4(b) shows the recovered B-mode image in the multi-channel scenario. It shows that the multi-channel scenario leads to a nearly perfect reconstrucion, with a peak-signal-to-noiseratio (PSNR), calculated against the reference image, of $28.3 \mathrm{~dB}$ while the 1-channel scenario leads to significantly lower image quality $(\mathrm{PSNR}=25.5 \mathrm{~dB})^{1}$.

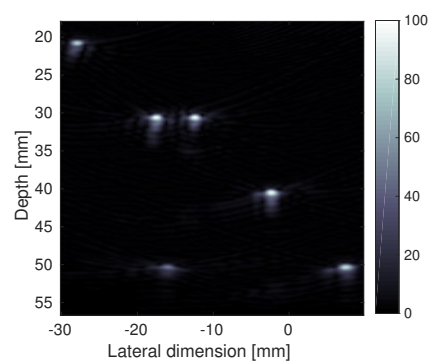

(a)

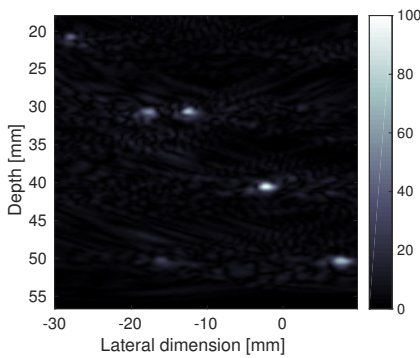

(b)
Fig. 4. (a) Original B-mode image; (b) Recovered B-mode image from $3 \%$ measurements in a 2-channels scenario $(P S N R=28.3 \mathrm{~dB})$.

\subsection{Limitations of the proposed method}

The current method requires a perfect estimation of the support in order to perform efficient reconstructions which is very difficult to achieve in real scenarios. To address this problem, the nonuniform sparse framework [12] will be explored in a future work. Another limitation of the current approach is the perfect knowledge of the pulse that can be tackled by exploring blind-deconvolution approaches $[1,13,14]$.

\section{CONCLUSION}

We have presented an extension of the pulse-stream model coined as multi-channel pulse-stream model. It accounts for the inter-sensor dependencies as an additional structure to the general pulse-stream model and enables us to quantitatively estimate the number of random projections necessary to sample such signals. We also suggest a reconstruction method based on $\ell_{1}$-minimization on the reduced signal support and illustrates its benefits on synthetic and experimental non-destructive-evaluation signals.

\footnotetext{
$1_{\text {https: //github.com/AdriBesson/ICASSP 2018-pulse-streams }}$
} 


\section{REFERENCES}

[1] Chinmay Hegde and Richard G. Baraniuk, "Sampling and Recovery of Pulse Streams," IEEE Trans. Signal Process., vol. 59, no. 4, pp. 1505-1517, apr 2011.

[2] Tanya Chernyakova and Yonina C. Eldar, "Fourier-domain Beamforming: the Path to Compressed Ultrasound Imaging," IEEE Trans. Ultrason. Ferroelectr. Freq. Control, vol. 61, no. 8, pp. 1252-1267, aug 2014.

[3] Ronen Tur, Yonina C. Eldar, and Zvi Friedman, "Innovation Rate Sampling of Pulse Streams With Application to Ultrasound Imaging," IEEE Trans. Signal Process., vol. 59, no. 4, pp. 1827-1842, apr 2011.

[4] Tamir Bendory, Avinoam Bar-Zion, Dan Adam, Shai Dekel, and Arie Feuer, "Stable Support Recovery of Stream of Pulses With Application to Ultrasound Imaging," IEEE Trans. Signal Process., vol. 64, no. 14, pp. 3750-3759, jul 2016.

[5] Ewen Carcreff, Sébastien Bourguignon, Jérôme Idier, and Laurent Simon, "A Linear Model Approach for Ultrasonic Inverse Problems with Attenuation and Dispersion," IEEE Trans. Ultrason. Ferroelectr. Freq. Control, vol. 61, no. 7, pp. 11911203, jul 2014.

[6] Omer Bar-Ilan and Yonina C. Eldar, "Sub-Nyquist Radar via Doppler Focusing," IEEE Trans. Signal Process., vol. 62, no. 7, pp. 1796-1811, apr 2014.

[7] Richard Baraniuk and Philippe Steeghs, "Compressive Radar Imaging," in IEEE Natl. Radar Conf. - Proc., apr 2007, pp. $128-133$.

[8] Richard G. Baraniuk, Volkan Cevher, Marco F. Duarte, and Chinmay Hegde, "Model-based Compressive Sensing," IEEE Trans. Inf. Theory, vol. 56, no. 4, pp. 1982-2001, apr 2010.

[9] Emmanuel J Candes, Yonina C Eldar, Deanna Needell, and Paige Randall, "Compressed Sensing with Coherent and Redundant Dictionaries," Appl. Comput. Harmon. Anal., vol. 31, no. 1, pp. 59-73, jul 2011.

[10] Stephen Boyd, "Distributed Optimization and Statistical Learning via the Alternating Direction Method of Multipliers," Found. Trends Mach. Learn., vol. 3, no. 1, pp. 1-122, jan 2011.

[11] Gabriel Montaldo, Mickaël Tanter, Jérémy Bercoff, Nicolas Benech, and Mathias Fink, "Coherent Plane-wave Compounding for Very High Frame Rate Ultrasonography and Transient Elastography," IEEE Trans. Ultrason. Ferroelectr. Freq. Control, vol. 56, no. 3, pp. 489-506, mar 2009.

[12] M. Amin Khajehnejad, Weiyu Xu, A. Salman Avestimehr, and Babak Hassibi, "Analyzing Weighted $\ell_{1}$ Minimization for Sparse Recovery with Nonuniform Sparse Models," IEEE Trans. Signal Process., vol. 59, no. 5, pp. 1985-2001, may 2011.

[13] Ramazan Demirli and Jafar Saniie, "Model-based Estimation of Ultrasonic Echoes Part I: Analysis and Algorithms," IEEE Trans. Ultrason. Ferroelectr. Freq. Control, vol. 48, no. 3, pp. 787-802, may 2001.

[14] Ningning Zhao, Qi Wei, Adrian Basarab, Denis Kouamé, and Jean-Yves Tourneret, "Blind Deconvolution of Medical Ultrasound Images Using a Parametric Model for the Point Spread Function," in 2016 IEEE Int. Ultrason. Symp., sep 2016, pp. $1-4$. 